\title{
Benutzervorteile rechtlich möglich
}

\section{Einschränkungen für "Dreckschleudern" und "Krachmacher" sowie Vorteile für geräuscharme und umweltschonende Fahrzeuge: Welcher engagierte Kommunal- politiker wünscht sich nicht solche Steuerungsmöglichkeiten für die Verbesse- rung der Attraktivität der Innenstädte oder den Schutz sensibler Wohnberei- che? Die Rahmenbedingungen dafür sind jetzł endlich geklärt.}

$\mathrm{U}$ Von Wulf-Holger Arndt mweltschonendere Fahrzeugtechniken, wie z.B. Gasfahrzeuge, sind seit einiger Zeit serienmäßig im Einsatz. Der fehlende Rahmen auf Bundesebene (Stichwort: ökologische Steuerreform) indes bremst die Durchsetzung dieser Antriebsarten. Auf der Ebene der Städte und Gemeinden herrscht noch relative Unsicherheit, inwieweit kommunal eine Förderung dieser Fahrzeuge möglich ist. Oft werden solche Maßnahmen nur mit Ausnahmegenehmigungen, zeitlich befristet oder eben gar nicht umgesetzt. Im Rahmen eines Forschungsvorhabens am IÖW ist jetzt erstmalig der rechtliche Rahmen für solche Maßnahmen geklärt worden (1). Daraus ergibt sich ein anwendungsorientierter Handlungsrabmen für die Förderung umweltverträglicheren Verkehrs und die notwendige planerische Einbettung der vorgesehenen Maß- nahmen.

Für deren Durchsetzung ist das Aufzeigen eines Begründungszusammenhanges für die Benutzervorteile eine wichtige Voraussetzung. Insbesondere wenn in bestehende Rechte und Verkehrsabläufe erheblich eingegriffen wird, wie beispielsweise bei einer flächenhaften Verkehrsberuhigung, ergeben sich besondere Rechtmäßigkeitsanforderungen, die im Laufe der weiteren Planungen zu erfüllen sind.

Im einzelnen sind die Belange der Betroffenen zu ermitteln und das Konzept für die Verkehrsmaßnahmen umfassend städtebaulich und verkehrsplanerisch einzubinden. Dies bedeutet, daß in der kommunalen Planung Qualitätsziele beschlossen sein müssen: etwa Maximalwerte für Verkehrsmengen im Verkehrsentwicklungsplan oder Zielwerte im Lärmminderungsplan. Der Begründungszusammenhang ergibt sich dann, wenn nachgewiesen wird, daß die Durchsetzung der Ziele über die Förderung umweltschonender Fahrzeuge erreichbar ist.

Ein geeignetes Verkehrsmaßnahmen-Konzept sollte also: wesentlich auch städtebaulich begründet werden (mit Formulierung von Qualitätszielen und einer Bestandsaufnahme),

- von dem für die städtebauliche Planung zuständigen Beschlußgremium beschlossen werden,

- insbesondere auch die Verlagerungswirkungen des Konzepts genauer betrachten und abwägen und

- eine sorgfältige Abwägung der betroffenen Belange vornehmen.

\section{Breites Maßnahmenspektrum}

Die Kommune sollte Vorschläge für Maßnahmen erarbeiten, die auf eine Belastungsreduzierung (Lärmminderung, Schadstoffreduktion usw.) gerichtet sind. Maßgeblich für die Auswahl sind dabei Gesichtspunkte der Entlastungswirkung, der rechtlichen und der tatsächlichen Durchsetzbarkeit. In den Modellstädten und -regionen des erwähnten Forschungsvorhabens (Augsburg, Wernigerode und die Insel Usedom) sind das z.B. die verkehrsberuhigte Innenstadt, Ausnahmen von Verkehrsbeschränkungen für schadstoff- und geräuscharme Fahrzeuge oder die vertraglich vereinbarte Transportkoordinierung (City-Logistik) der ansiedelnden Unternehmen im Güterverkehrszentrum.

Das mögliche Spektrum an Maßnahmen zieht sich von der Fahrzeugtechnik über die Straßenraumgestaltung oder verkehrsregelnde Maßnahmen nach $\S 45$ StVO bis zur Standortplanung und zum Städtebau. Für die erfolgreiche Umsetzung der Benutzervorteile muß ein Optimum zwischen (möglichst großer) Wirkungstiefe und (möglichst geringer) Eingriffstiefe gesucht werden. Falls die Wirkungen einer Maßnahme nicht hinreichend abgeschätzt werden können, muß deshalb nicht auf die Umsetzung verzichtet werden. Mittels einer "Versuchs-Anordnung“ können die Folgen erprobt werden.
Das Rechtsgutachten weist nach, daß die Gewährung von Benutzervorteilen nicht nur für Fahrzeuge mit emissionsarmer Antriebstechnik, sondern auch für Fahrzeuge mit einer umweltschonenden Transporttechnologie (City-Logistik) möglich ist. Es genügt ein sachlicher Anknüpfungspunkt und das Überwiegen des öffentlichen Interesses an der Maßnahme im Rahmen einer Interessenabwägung. Der sachliche Anknüpfungspunkt ist dabei i.d.R. der umweltbezogene Vorteil der Fahrzeuggruppen oder - anders herum - die spezifische Gruppenverantwortung der ausgeschlossenen Fahrzeuggruppen für die Lärm- und Abgasbelastung, der begegnet werden soll. Die Verhältnismäßigkeit der Maßnahmen sollte weiterhin durch die Festsetzung von zeitlich begrenzten Übergangs- und in begründeten Fällen Ausnahmeregelungen gesichert werden.

Sinnvoll für die Umsetzung der Maßnahmen erscheint ein Stufenkonzept. Werden die vorgesehenen Maßnahmen zur Verbesserung der urbanen Umwelt durch die kommunalen Entscheidungsträger wie beschrieben flankiert, ist ein wichtiger Schritt zu ihrer erfolgreichen Verwirklichung getan.

\section{Anmerkung}

(1) Die Studie "Erprobung von Maßnahmen zur umweltschonenden Abwicklung des städtischen Wirtschoftsverkehrs" wird vom IÖW im Rahmen des Umwelfforschungsplans 1996 des Umwelthundesamtes durchgeführt. Dabei werden Möglichkeiten und Wirkungen der Förderung des Gasantriebs als besonders emissionsarmer Antrieb und der City-Logistik als Maßnahme zur Vermeidung von innerstädtischem Wirtschaftsverkehr untersucht. Zu den Bausteinen gehören ein „Rechtsgutachten zu Benutzervorteilen für gasbetriebene und City-Logistik-Fahrzeuge" und ein "Handlungsrahmen für Maßnahmen zur Umweltentlastung im städtischen Wirtschaftsverkehr". Für eine ausführlichere Darstellung des Gesamtprojekts vgl. W.H. Anndt: Modellhafte Maßnahmen, in: Ökologisches Wirtschaften 6/1997, S. 29-30.

\section{Der Autor}

Wulf-Holger Arndt ist wissenschaftlicher Mitarbeiter im Forschungsfeld Stadtentwicklung, Planung und Verkehr des IÖW.

Kontakt: IÖW, Giesebrechtstr. 13, 10629 Berlin, Tel. 030/88 45 94-0, Fax 030/88 25 439, E-mail: wulf-holger.arndł@ioew.b.eunet.de 
(c) 20I0 Authors; licensee IÖW and oekom verlag. This is an article distributed under the terms of the Creative Commons Attribution Non-Commercial No Derivates License (http://creativecommons.org/licenses/by-nc-nd/3.o/), which permits unrestricted use, distribution, and reproduction in any medium, provided the original work is properly cited. 